\title{
Procalcitonin reflects bacteremia and bacterial load in urosepsis syndrome: a prospective observational study
}

\author{
Cees van Nieuwkoop ${ }^{1 *}$, Tobias N Bonten ${ }^{1}$, Jan W van't Wout ${ }^{1,2}$, Ed J Kuijper ${ }^{3}$, Geert H Groeneveld ${ }^{4}$, \\ Martin J Becker ${ }^{5}$, Ted Koster ${ }^{6}$, G Hanke Wattel-Louis ${ }^{7}$, Nathalie M Delfos ${ }^{8}$, Hans C Ablij ${ }^{9}$, Eliane MS Leyten ${ }^{4}$, \\ Jaap $T$ van Dissel ${ }^{1}$
}

\begin{abstract}
Introduction: Guidelines recommend that two blood cultures be performed in patients with febrile urinary tract infection (UTI), to detect bacteremia and help diagnose urosepsis. The usefulness and cost-effectiveness of this practice have been criticized. This study aimed to evaluate clinical characteristics and the biomarker procalcitonin (PCT) as an aid in predicting bacteremia.
\end{abstract}

Methods: A prospective observational multicenter cohort study included consecutive adults with febrile UTI in 35 primary care units and 8 emergency departments of 7 regional hospitals. Clinical and microbiological data were collected and PCT and time to positivity (TTP) of blood culture were measured.

Results: Of 581 evaluable patients, 136 (23\%) had bacteremia. The median age was 66 years (interquartile range 46 to 78 years) and 219 (38\%) were male. We evaluated three different models: a clinical model including seven bedside characteristics, the clinical model plus PCT, and a PCT only model. The diagnostic abilities of these models as reflected by area under the curve of the receiver operating characteristic were 0.71 ( $95 \%$ confidence interval (Cl): 0.66 to 0.76$), 0.79$ ( $95 \%$ Cl: 0.75 to 0.83 ) and 0.73 (95\% Cl: 0.68 to 0.77 ) respectively. Calculating corresponding sensitivity and specificity for the presence of bacteremia after each step of adding a significant predictor in the model yielded that the PCT $>0.25 \mu \mathrm{g} / \mathrm{l}$ only model had the best diagnostic performance (sensitivity $0.95 ; 95 \% \mathrm{Cl}$ : 0.89 to 0.98 , specificity $0.50 ; 95 \% \mathrm{Cl}: 0.46$ to 0.55 ). Using PCT as a single decision tool, this would result in $40 \%$ fewer blood cultures being taken, while still identifying 94 to $99 \%$ of patients with bacteremia.

The TTP of E. coli positive blood cultures was linearly correlated with the PCT log value; the higher the PCT the shorter the TTP $\left(R^{2}=0.278, P=0.007\right)$.

Conclusions: PCT accurately predicts the presence of bacteremia and bacterial load in patients with febrile UTI. This may be a helpful biomarker to limit use of blood culture resources.

\section{Introduction}

Urinary tract infection (UTI) is one of the most common infectious diseases. Fever in UTI typically represents the presence of acute pyelonephritis but it may also reflect prostatitis and/or the urosepsis syndrome $[1,2]$. Patients with febrile UTI generally present with mild illness in primary care but may rapidly develop a

\footnotetext{
* Correspondence: c.van_nieuwkoop@lumc.nl

'Department of Infectious Diseases, Leiden University Medical Center

Albinusdreef 2, 2333 ZA, Leiden, The Netherlands

Full list of author information is available at the end of the article
}

life-threatening condition, progressing into septic shock and multiple organ failure. The overall mortality rate of pyelonephritis is approximately $0.3 \%$, but in bacteremic patients it can be as high as $7.5 \%$ to $30 \%$ [3,4]. In addition, bacteremia in UTI is associated with prolonged hospitalization and higher complication rates [5-7]. Given this spectrum of disease, clinicians are vigilant to identify bacteremia at a patient's presentation.

The incidence of bacteremia in patients with acute pyelonephritis has been reported to be roughly $20 \%$ [8-10]. Several studies have been conducted to identify

\section{() Biomed Central}


predictive characteristics of bacteremia in patients with UTI $[6,7,11,12]$. However, no single clinical model has been used in practice because of its poor value in predicting bacteremia. The gold standard for detection of bacteremia remains the performance of at least two blood cultures to achieve sufficient sensitivity [13]. There are, however, practical limitations. First of all, it takes at least 24 to 48 hours to attain the culture result. Secondly, there may be a false positive result as contamination rates of up to $7 \%$ have been reported [14]. Furthermore, the implementation of the surviving sepsis campaign, which recommends the immediate initiation of broad-spectrum antibiotic therapy once septicemia is suspected, leads to an increase in the performance of blood cultures with lower yield, likely reflecting the obtainment of additional cultures after initiation of antibiotics $[15,16]$. Therefore, there is a need for strategies that guide clinicians and help reduce avoidable blood cultures and, by consequence, medical costs.

The biomarker procalcitonin (PCT) is a marker of systemic inflammation and thus it may help to predict bacteremia $[17,18]$. The aim of this study was to assess clinical characteristics and the PCT value to predict bacteremia in patients with febrile UTI.

\section{Materials and methods}

\section{Study design and setting}

We conducted a prospective observational multicenter cohort study. Eight emergency departments (ED) of 7 hospitals and 35 affiliating primary health care centers, serving one single area of the Netherlands, participated. Consecutive patients who presented with a diagnosis of febrile UTI, were considered for enrollment in the study. Recruitment took place from January 2004 through November 2008 but each centre started at different time points. The study was approved by the local ethics committees and all included patients gave written informed consent.

\section{Inclusion and exclusion criteria}

Inclusion criteria were: age of 18 years or above, fever (defined as an tympanic temperature $\geq 38.0^{\circ} \mathrm{C}$ or a history of fever and chills within 24 hours before presentation), at least one symptom of UTI (dysuria, frequency, urgency, perineal pain, flank pain or costovertebral tenderness) and a positive nitrite dipstick test or leukocyturia as defined by a positive leukocyte esterase dipstick test or the presence of more than five leukocytes per high-power field in a centrifuged sediment. Exclusion criteria were current treatment for urolithiasis or hydronephrosis, pregnancy, hemo- or peritoneal dialysis, a history of kidney transplantation or known presence of polycystic kidney disease.

\section{Procedures and definitions}

Clinical data and laboratory values were collected by qualified research nurses or the clinical investigators (CvN, TNB). Baseline data were collected within 24 hours of enrolment by a standardized questionnaire of the patient and reviewing the medical record. All patients were empirically treated with antibiotics according to local policy (oral ciprofloxacin $500 \mathrm{mg}$ twice daily for outpatients and cefuroxim \pm gentamicin intravenously for inpatients). Based on the culture results, hospitalized patients were subsequently switched to oral antibiotic treatment (first choice ciprofloxacin).

Blood cultures were obtained before commencement of antimicrobial therapy and were analyzed using local standard microbiological methods. At least two sets of $10 \mathrm{~mL}$ blood samples were taken and inoculated into aerobic bottles, which were incubated into an automated continuous monitoring system. In the Leiden University Medical Center (LUMC), the BACTEC 9240 (Becton Dickinson Diagnostic Instrument Systems, Sparks, MD, USA) was used, which monitors $\mathrm{CO}_{2}$ production every 10 minutes by means of a fluorescent signal. The bottles were loaded in the automated system once received at the laboratory. The time to positivity (TTP), defined as the time from the start of incubation to the start of the alert signal (as documented by the monitoring system), was recorded for each bottle of positive blood cultures. When multiple cultures were positive, the shortest TTP was selected for analysis. TTP was analyzed for E. coli positive blood cultures and confined to results in one center, the LUMC, as the TTP depends on the microorganism and the logistics of blood culture performance (for example, transport time from blood culture obtainment to incubator) [19].

Clean midstream-catch urine cultures were obtained before starting antimicrobial therapy and were analyzed using local standard microbiological methods. In case of a urinary catheter, the urine sample was collected from the port of the catheter. A positive urine culture was defined as bacterial growth over $10^{3} \mathrm{CFU} / \mathrm{ml}$ urine or a bacterial monoculture over $10^{2} \mathrm{CFU} / \mathrm{ml}$ urine in the presence of pyuria [20]. Urine cultures revealing growth of two or more different bacterial species reflecting mixed skin or gut flora were considered to indicate contamination [20].

Plasma EDTA blood samples were collected, centrifuged and stored at $-80^{\circ} \mathrm{C}$ within two hours of patient enrolment. PCT levels were measured after the completion of all study enrolments, using a Time Resolved Amplified Cryptate Emission technology assay (TRACE ${ }^{\oplus}$, Kryptor compact, PCTsensitive; Brahms AG; Hennigsdorf, Germany).

Bacteremia was defined as growth of any pathogen in the blood culture. The isolation of coagulase-negative 
staphylococci from the blood culture was considered to indicate contamination and thus absence of bacteremia.

\section{Statistical analysis}

Descriptive analysis included means or percentages with 95\% confidence intervals (CIs) or medians and ranges, as appropriate. Missing values of categorical variables were considered to indicate the absence of that characteristic. This was applied for shaking chills $(n=66)$ and costovertebral tenderness $(n=18)$. Univariate analysis was performed using the Student's $t$-test or Mann-Whitney $U$ test for continuous variables and Chi-square tests for categorical variables. Covariates found to be associated with bacteremia on univariate analysis at a level of significance $P<0.2$ were eligible for inclusion in a multivariate logistic regression model using a backward selection procedure [21]. Measures for association were expressed as odds ratios (ORs) for disease with their 95\% CIs for categorical variables. We tested the following three models: 1). A clinical model including clinical variables only; 2). A clinical model added with the PCT value; 3$)$. A model based on PCT only. The predicted probabilities of bacteremia $\left(P_{\text {bac }}\right)$ in any patient for the different models were calculated by using the following regression equation: $\ln \left(P_{\text {bac }} /\left(1-P_{\text {bac }}\right)\right)=$ intercept $+\beta$ coefficient * variable, where the intercept and $\beta$-coefficient are obtained from logistic regression analysis. We constructed receiver operating characteristic (ROC)curves for the different models using $P_{\text {bac }}$ as the test variable and bacteremia (yes/no) as state variable. The discriminative power and the diagnostic performance of the prediction models were compared by calculating the area under the curve (AUC) of the ROC-curve and by Nagelkerke's $R^{2}$. In addition, for the clinical models, based on the $\beta$-coefficient, points were assigned for each predictor and different cutoff values were used to calculate corresponding sensitivity, specificity, positive and negative predictive values (PPV, NPV) and likelihood ratios for predicting bacteremia were calculated. For PCT, different cutoff values were tested, according to the instructions by the manufacturer for diagnosis of bacterial sepsis or lower respiratory tract infection; the cutoff value corresponding with a sensitivity of $95 \%$ and highest specificity was chosen for further analysis. A $P$ value $<0.05$ was considered indicative for statistical significance. SPSS software (SPSS Inc., Chicago, Ill, USA; version 17.0) was used for statistical analysis.

\section{Results}

\section{Patient characteristics and microbiological results}

Of 728 patients screened for eligibility, 642 met the inclusion criteria and were included in the study of which 581 were evaluable with concurrent blood cultures and PCT measurements at baseline. Patients excluded from analysis because of missing blood culture or PCT value, were similar with respect to demographics and clinical features. The majority (75\%) presented at EDs. The median age was 66 years, 38\% were men and $52 \%$ had co-existing illnesses. Details of the baseline characteristics are listed in Table 1.

Bacteremia was present in 131 (23\%) patients: Escherichia coli, $n=104,(79 \%)$; Klebsiella spp., $n=6$, (5\%); Proteus spp., $n=5$ (4\%), Pseudomonas aeroginosa, $n=3$ (2\%); Staphylococcus aureus, $n=2$ (2\%); Enterococcus spp., $n=2(2 \%)$; and other, $n=9(7 \%)$. Only sixteen patients (3\%) had coagulase-negative staphylococci in their blood culture; this was considered contamination. Bacteremic patients were significantly older, they significantly had more diabetes mellitus, shaking chills, or were pretreated for UTI; costovertebral tenderness was significantly less frequently present. On physical examination bacteremic patients more frequently had altered mental status, and they significantly had higher temperature and heart rate (Table 1).

Urine cultures were done in 559 (96\%) patients and revealed the following: E. coli, $n=319$ (57\%); Klebsiella spp., $n=25$ (4\%); Pseudomonas aeroginosa, $n=14(3 \%)$; Proteus spp., $n=12$ (2\%); Enterococcus spp., $n=10$ (2\%); Staphylococcus spp., $n=12(2 \%)$; other uropathogen, $n=21$ (3\%); contaminated, $n=77(14 \%)$ and negative urine culture, $n=71(13 \%)$. In those patients of whom no definite uropathogen was isolated, $69 \%$ had antibiotic UTI treatment during obtainment of the urine culture sample.

Results of con- and discordant blood and urine cultures have been described previously [22].

\section{Procalcitonin and microbiological outcome}

The AUC of the ROC-curve of PCT diagnosing bacteremia was 0.81 (95\% CI: 0.77 to 0.85 ) indicating good discriminative power (Figure 1). The corresponding sensitivity, specificity, NPV, PPV and likelihood ratios of different PCT cutoff values are also outlined in Figure 1. A cutoff value $>0.25 \mu \mathrm{g} / \mathrm{l}$ had a sensitivity of $95 \%$ and was chosen for further analysis in prediction modeling.

As the predictive value of PCT might have been influenced by the antibiotic treatment at the time of presentation in $29 \%$ of the patients, the analysis was also done separately. In patients on active antibiotic UTI treatment, bacteremia was present in $29 \%$ of the cases compared to $20 \%$ in those without antibiotic treatment. Corresponding AUC of the ROC-curve were 0.83 (95\% CI: 0.76 to 0.89 ) and 0.80 (95\% CI: 0.75 to 0.85 ), respectively, indicating that antibiotic treatment did not alter the predictive value of $\mathrm{PCT}$ with respect to bacteremia.

As undetectable PCT levels may be indicative of absence of bacterial infection we additionally tested whether a PCT value $<0.06 \mu \mathrm{g} / \mathrm{l}$ was correlated with a 
Table 1 Baseline characteristics of 581 patients presenting with febrile UTI.

\begin{tabular}{|c|c|c|c|c|}
\hline Characteristic at presentation & $\begin{array}{l}\text { All patients } \\
n=581\end{array}$ & $\begin{array}{c}\text { Non-bacteremic } \\
n=450\end{array}$ & $\begin{array}{c}\text { Bacteremic } \\
n=131\end{array}$ & $P$ \\
\hline \multicolumn{5}{|l|}{ Demographics } \\
\hline Age, years, median (IQR) & 66 (46 to 78$)$ & 63 (42 to 77 ) & 74 (60 to 84$)$ & $<0.001$ \\
\hline Male sex & $219(38)$ & $163(36)$ & $56(43)$ & 0.175 \\
\hline Nursing home residency & $29(5)$ & $20(4)$ & $9(7)$ & 0.262 \\
\hline \multicolumn{5}{|l|}{ Co-morbidity } \\
\hline Any & $301(52)$ & $226(50)$ & $75(57)$ & 0.156 \\
\hline Diabetes mellitus & $96(17)$ & $65(14)$ & $31(24)$ & 0.012 \\
\hline Malignancy ${ }^{\mathrm{a}}$ & $63(11)$ & $44(10)$ & $19(15)$ & 0.126 \\
\hline Urinary catheter ${ }^{b}$ & $45(8)$ & $30(7)$ & $15(12)$ & 0.093 \\
\hline Urinary tract disorder $^{c}$ & $144(25)$ & $110(24)$ & $34(26)$ & 0.725 \\
\hline Immunocompromised & $87(15)$ & $68(15)$ & $19(15)$ & 0.864 \\
\hline \multicolumn{5}{|l|}{ History, Signs and Symptoms } \\
\hline Antibiotic UTI treatment ${ }^{d}$ & $167(29)$ & $119(26)$ & $48(37)$ & 0.023 \\
\hline Fever duration, hours, median (IQR) & 24 (12 to 53$)$ & 24 (12 to 48$)$ & 24 (12 to 72$)$ & 0.791 \\
\hline Altered mental status & $46(8)$ & $27(6)$ & $19(15)$ & 0.002 \\
\hline Shaking chills & $320(55)$ & $236(52)$ & $84(64)$ & 0.018 \\
\hline Costovertebral tenderness & $352(61)$ & $283(63)$ & $69(53)$ & 0.035 \\
\hline Temperature, ${ }^{\circ} \mathrm{C}$, mean $\pm \mathrm{SD}$ & $38.6 \pm 1.05$ & $38.5 \pm 1.06$ & $38.8 \pm 0.99$ & 0.001 \\
\hline $\mathrm{MAP}, \mathrm{mmHg}$, mean $\pm \mathrm{SD}$ & $101 \pm 17$ & $102 \pm 17$ & $100 \pm 19$ & 0.479 \\
\hline $\mathrm{HR}$, beats/minute, mean $\pm \mathrm{SD}$ & $93 \pm 18$ & $91 \pm 17$ & $98 \pm 20$ & $<0.001$ \\
\hline $\mathrm{PCT}, \mu \mathrm{g} / \mathrm{L}$, median $(\mathrm{IQR})$ & 0.41 (0.13 to 1.68$)$ & 0.25 (0.10 to 0.90$)$ & 2.29 (0.72 to 9.07$)$ & $<0.001$ \\
\hline
\end{tabular}

Data are presented as $n(\%)$ unless otherwise stated. IQR, interquartile range; SD, standard deviation; MAP, mean arterial pressure; HR, heart rate; PCT, procalcitonin. ${ }^{a}$ Defined as any cancer except basal- or squamous-cell cancer of the skin that was active within the previous year. ${ }^{b}$ Indwelling urethral catheter ( $n$ $=36)$, supra-pubic catheter $(n=7)$, intermittent urethral self-catherization $(n=2)$. ${ }^{c}$ Defined as the presence of any functional or anatomical abnormality of the urinary tract. ${ }^{\mathrm{d}}$ Present oral antibiotic treatment for non-febrile UTI (that is, fever developed during UTI treatment). ${ }^{\text {e }}$ Missing value in 81 patients.

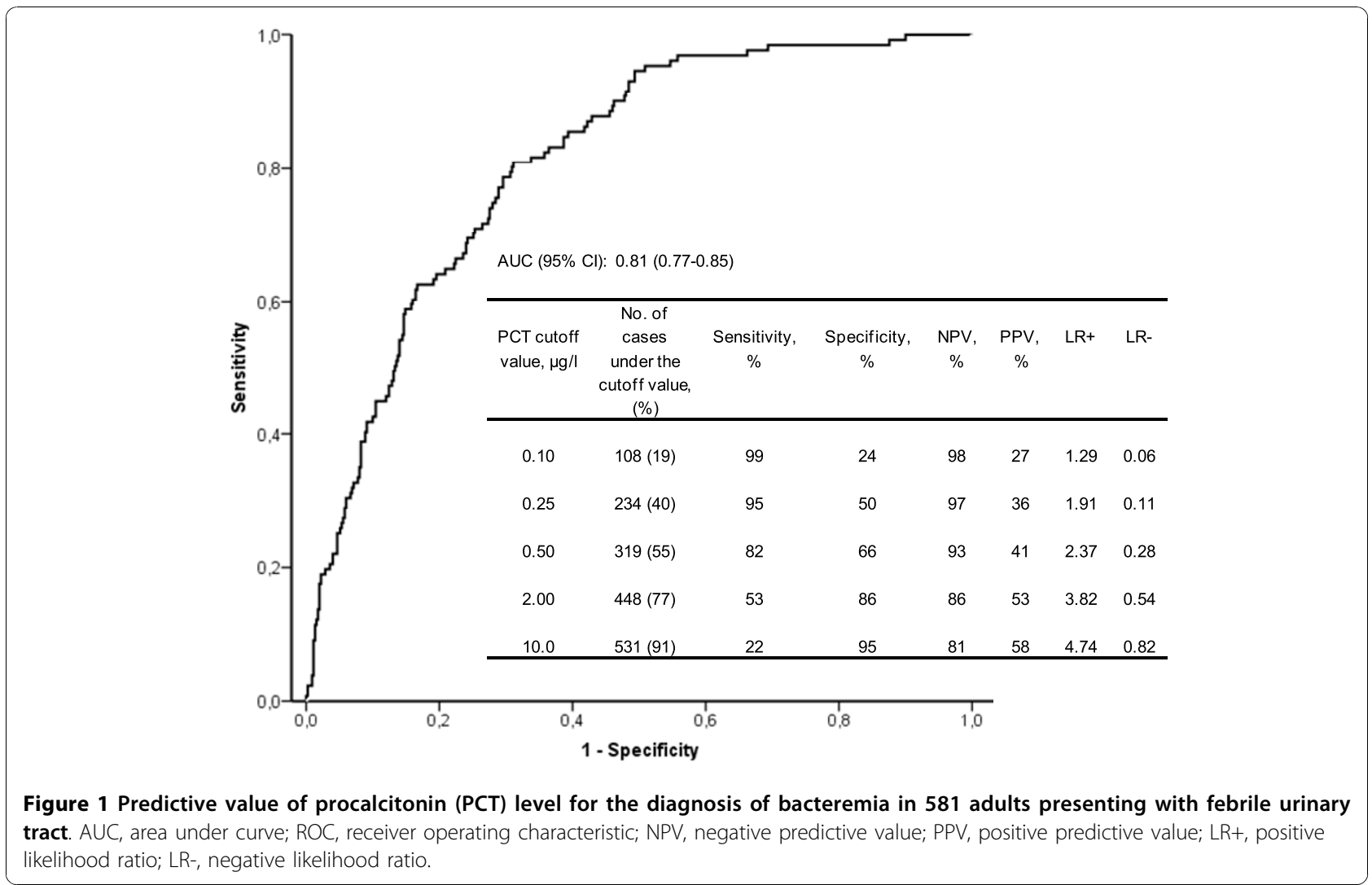


negative urine culture. Indeed a PCT $<0.06 \mu \mathrm{g} / \mathrm{l}$ was associated with a lower rate of negative urine cultures, $11 \%$ versus $13 \%$ for $\mathrm{PCT} \geq 0.06 \mu \mathrm{g} / \mathrm{l}$, but this difference was not statistically significant (OR $0.8 ; 95 \%$ CI: 0.3 to 2.2, $P=0.821$ ).

\section{Predictors of bacteremia}

Clinical variables that were found to have an association with the presence of bacteremia with a $P$-value $<0.2$ were entered as covariates into a multivariate logistic regression model. Then PCT $>0.25 \mu \mathrm{g} / \mathrm{l}$ was added as a variable in a second model and finally a univariate model of PCT $>0.25 \mu \mathrm{g} / \mathrm{l}$ was tested. This resulted in three different models (model 1, 2 and 3 respectively) as shown in Table 2. Older age, higher temperature and heart rate were significantly associated with bacteremia in the clinical model 1. When PCT was added to this clinical model (model 2), PCT appeared to be the strongest predictor (OR 14.7) for bacteremia, besides the significant clinical predictors temperature $>38.6^{\circ} \mathrm{C}$ (OR 1.7) and diabetes mellitus (OR 1.8). The discriminative ability of model 2 with respect to Nagelkerke's $R^{2}$ was much better than the clinical model 1 (0.293 vs 0.145 ) but comparable with model 3 based on PCT only (0.252).

\section{Diagnostic value of prediction models}

For each model we calculated the probability of bacteremia $\left(P_{\text {bac }}\right)$ for every individual patient with the equation

Table 2 Multivariate logistic regression models predicting bacteremia in $\mathbf{5 8 1}$ patients with febrile UTI.

\begin{tabular}{|c|c|c|c|}
\hline & $\begin{array}{c}\text { Multivariate OR } \\
(95 \% \mathrm{Cl})\end{array}$ & $P$-value & $\mathrm{R}^{2}$ \\
\hline Model 1 & & & 0.145 \\
\hline Age $>65$ years & $2.4(1.5$ to 3.8$)$ & $<0.001$ & \\
\hline Temperature $>38.6^{\circ} \mathrm{C}$ & 2.1 (1.3 to 3.3$)$ & 0.001 & \\
\hline Altered mental status & 1.8 (0.9 to 3.5$)$ & 0.093 & \\
\hline Heart rate $>100 /$ minute & 1.7 (1.1 to 2.7$)$ & 0.015 & \\
\hline Diabetes mellitus & 1.6 (1.0 to 2.7) & 0.063 & \\
\hline Shaking chills & 1.5 (1.0 to 2.3$)$ & 0.052 & \\
\hline Antibiotic UTI treatment & 1.5 (0.9 to 2.3) & 0.085 & \\
\hline Model 2 & & & 0.293 \\
\hline Age $>65$ years & 1.6 (1.0 to 2.5$)$ & 0.059 & \\
\hline Temperature $>38.6^{\circ} \mathrm{C}$ & 1.7 (1.1 to 2.7 ) & 0.019 & \\
\hline Altered mental status & 2.0 (1.0 to 4.2$)$ & 0.054 & \\
\hline Diabetes mellitus & $1.8(1.0$ to 3.1$)$ & 0.035 & \\
\hline PCT > $0.25 \mu \mathrm{g} / \mathrm{l}$ & 14.7 (6.6 to 32.6$)$ & $<0.001$ & \\
\hline Model 3 (univariate) & & & 0.252 \\
\hline $\mathrm{PCT}>0.25 \mu \mathrm{g} / \mathrm{l}$ & $18.0(8.2$ to 39.5$)$ & $<0.001$ & \\
\hline
\end{tabular}

UTI: urinary tract infection; OR: Odds Ratio; Cl: confidence interval; PCT: procalcitonin; $R^{2}$ : Nagelkerke's $R^{2}$.

Model $1=$ Clinical model including all clinical variables of Table 1 with $P$-value $<0.2$ in univariate analysis.

Model $2=$ Model $1+$ PCT $>0.25 \mu \mathrm{g} / \mathrm{l}$. Model $3=\mathrm{PCT}>0.25 \mu \mathrm{g} / \mathrm{l}$ only. as described above and compared the discriminative power of each model by constructing ROC-curves. Model 1, 2 and 3 had an AUC of ROC of 0.71 (95\% CI: 0.66 to 0.76$), 0.79$ (95\% CI: 0.75 to 0.83 ) and 0.73 (95\% CI: 0.67 to 0.77$)$, respectively.

In addition, we evaluated the diagnostic performance of each model in detecting bacteremia by measuring sensitivity, specificity, NPV, PPV and likelihood ratios. For model 1 and 2 we started with the most significant clinical predictor as indicated by the lowest $P$-value out of the multivariable analysis (Table 2) and then we stepwise added the next significant clinical predictor with increasing order of $P$-values. For each step, the corresponding sensitivity, specificity, NPV, PPV and likelihood ratios were calculated. In addition, the same was done in model 2 starting with PCT and then adding the clinical predictors. The results of this analysis are outlined in Table 3 . Only model 2 and 3 including PCT as a predictor had a NPV $>95 \%$ but model 3 (PCT $>0.25 \mu \mathrm{g} / \mathrm{l}$ only) had a better PPV. Thus the discriminative ability of PCT alone is better than PCT plus clinical predictors.

\section{Procalcitonin and time to positivity of blood culture}

The TTP was available in 25 of $26 \mathrm{E}$. coli positive blood cultures. The mean TTP was 11.6 hours (range 1.3 to $31.4 \mathrm{hrs}$ ). Plotting TTP with the log value of PCT resulted in a significant linear correlation $\left(\mathrm{R}^{2}=0.278, P\right.$ $=0.007)$, being the higher the PCT the shorter the TTP (Figure 2).

\section{Potential cost-savings of blood culture resources}

We calculated potential cost-savings assuming two sets of blood cultures will cost $\$ 140$ and the cost of PCT is $\$ 20$ per measurement. In this cohort, using a preset PCT cutoff value of $\leq 0.25 \mu \mathrm{g} / \mathrm{l}$ would save $40 \%$ of blood cultures while still identifying $97 \%$ of bacteremias. Thus the potential saving in blood culture resources is $(\$ 140$ times 0.40 minus $\$ 20$ ) $\$ 36$ per patient and $\$ 20.916$ for the whole cohort of 581 patients.

\section{Discussion}

In this study, we evaluated the ability of clinical and laboratory characteristics to predict bacteremia in adults presenting with febrile UTI. We found that PCT dichotomized around $0.25 \mu \mathrm{g} / \mathrm{l}$, is a robust surrogate marker for bacteremia, whereas the actual PCT value reflects bacterial load in the blood stream. PCT might be applied to help guide and limit the use of blood culture resources.

We used a PCT cutoff value of $\leq 0.25 \mu \mathrm{g} / \mathrm{l}$ after having tested different standard cutoff values as has been advocated by the manufacturer's instructions to indicate absence or presence of sepsis or even absence or 
Table 3 Predictive value of different models predicting bacteremia in $\mathbf{5 8 1}$ adults with febrile UTI.

\begin{tabular}{|c|c|c|c|c|c|c|}
\hline $\begin{array}{l}\text { No. patients } \\
\text { without } \\
\text { risk factor (\%) }\end{array}$ & $\begin{array}{c}\text { Sensitivity, } \\
\% \\
(95 \% \mathrm{Cl})\end{array}$ & $\begin{array}{c}\text { Specificity, } \\
\% \\
(95 \% \text { Cl) }\end{array}$ & $\begin{array}{l}\text { NPV, \% } \\
(95 \% \mathrm{Cl})\end{array}$ & $\begin{array}{l}\text { PPV, \% } \\
(95 \% \text { CI) }\end{array}$ & $\begin{array}{c}\mathrm{LR}+ \\
(95 \% \mathrm{Cl})\end{array}$ & $\begin{array}{c}\text { LR - } \\
(95 \% \mathrm{Cl})\end{array}$ \\
\hline
\end{tabular}

\section{Model 1}

Risk factor $A$

Risk factors $A, B$

Risk factors $A, B, C$

Model 2

Risk factor $-B$

Risk factors $B, D$

Risk factors $P, B$

Risk factors $P, B, D$

Model 3

PCT $>0.25 \mu \mathrm{g} / \mathrm{l}$

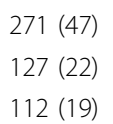

70 (62 to 78$) \quad 52$ (46 to 56$) \quad 86$ (81 to 89$) \quad 30$ (25 to 35$) \quad 1.45$ (1.25 to 1.68

90 (83 to 94$) 25$ (21 to 30$) \quad 90$ (83 to 94$) \quad 26$ (22 to 30$) \quad 1.21$ (1.12 to 1.30$)$

93 (87 to 97$) \quad 23$ (19 to 27$) \quad 92$ (85 to 96$) \quad 26$ (22 to 30$) \quad 1.21$ (1.13 to 1.29 )

68 (60 to 76$) \quad 51$ (46 to 56$) \quad 85$ (80 to 89$) \quad 29$ (24 to 34$) \quad 1.39$ (1.21 to 1.62 )

78 (70 to 85$) \quad 45$ (40 to 49$) \quad 88$ (83 to 92$) \quad 29$ (25 to 34$) \quad 1.42$ (1.26 to 1.61 )

97 (92 to 99) 30 (26 to 34) 97 (92 to 99) 29 (25 to 33) 1.39 (1.30 to 1.49)

97 (92 to 99$) \quad 25$ (21 to 29$) \quad 97$ (91 to 99$) \quad 27$ (23 to 32$) \quad 1.29$ (1.21 to 1.37$)$
$270(46)$

$229(39)$

140 (24)

$116(20)$
$0.58(0.44$ to 0.75$)$

$0.39(0.23$ to 0.66$)$

0.30 (0.16 to 0.57 )

95 (89 to 98) 50 (46 to 55) 97 (94 to 99) 36 (31 to 41) 1.91 (1.73 to 2.11) 0.11 (0.05 to 0.22 )

0.61 ( 0.48 to 0.80$)$

$0.48(0.34$ to 0.67$)$

0.10 (0.04 to 0.27$)$

0.12 (0.05 to 0.33 )

NPV, negative predictive value; PPV, positive predictive value; LR+, positive likelihood ration; LR-, negative likelihood ratio; $A$, Age $>65$ years; $B$, Temperature

$>38.6^{\circ} \mathrm{C}$; C, heart rate $>100$ /minute; D, diabetes mellitus; P, PCT $>0.25 \mu \mathrm{g} / \mathrm{l}$. For Model 1 and Model 2 the corresponding sensitivity, specificity, NPV, PPV, LR+ and LR- are calculated using a cutoff value of $\geq 1$ risk factor.

presence of bacterial infection as has previously been demonstrated in lower respiratory tract infections [23]. Compared to studies regarding PCT and bacteremia in infections other than febrile UTI, our diagnostic threshold was lower resulting in a higher sensitivity and lower specificity $[17,18,24,25]$. A recent study with similar design in patients presenting with community acquired pneumonia demonstrated highly similar findings [26]. In that study, a PCT value $\leq 0.25 \mu \mathrm{g} / \mathrm{l}$ would allow reducing blood cultures by $37 \%$ while still identifying $96 \%$ of bacteremias [26].

Using a PCT value $\leq 0.25 \mu \mathrm{g} / \mathrm{l}$, we demonstrate a $40 \%$ reduction of blood cultures in our study population while still identifying $97 \%$ of bacteremias. Using PCT as a decision rule to guide taking blood cultures in febrile UTI would thus likely to be cost-effective. Moreover, it might prevent false-positive blood cultures and costs of associated medical consultations. However, other laboratory values that might routinely be measured in patients presenting with febrile UTI such as C-reactive protein (CRP) and the erythrocyte sedimentation rate (ESR) could also be indicative for the presence of bacteremia. In this study, CRP and ESR were measured in a subset of ED patients when indicated by the attending physician. Both were significantly associated with bacteremia but had very limited diagnostic ability compared to PCT (see Additional file 1). This is like other studies that did not recommend the use of CRP and ESR for diagnosing bacteremia [24,25].

The clinical characteristics associated with the presence of bacteremia comprise two categories. One comprises clinical signs which are a result of the host's response to bacterial components and cytokines elicited by the local infection and possible systemic expansion (that is, chills, confusion, temperature $>38.6^{\circ} \mathrm{C}$, heart rate $>100 /$ minute) and the other category includes hostrelated risk factors for a complicated clinical course of disease such as older age and diabetes. All these clinical factors were found to be associated with bacteremia in previous studies in patients with UTI $[6,7,11,12]$. Similar to previous reports on smaller cohorts, we were not able to accurately predict the presence of bacteremia based on clinical characteristics only. Likely, this can be explained in part by the relatively old study population

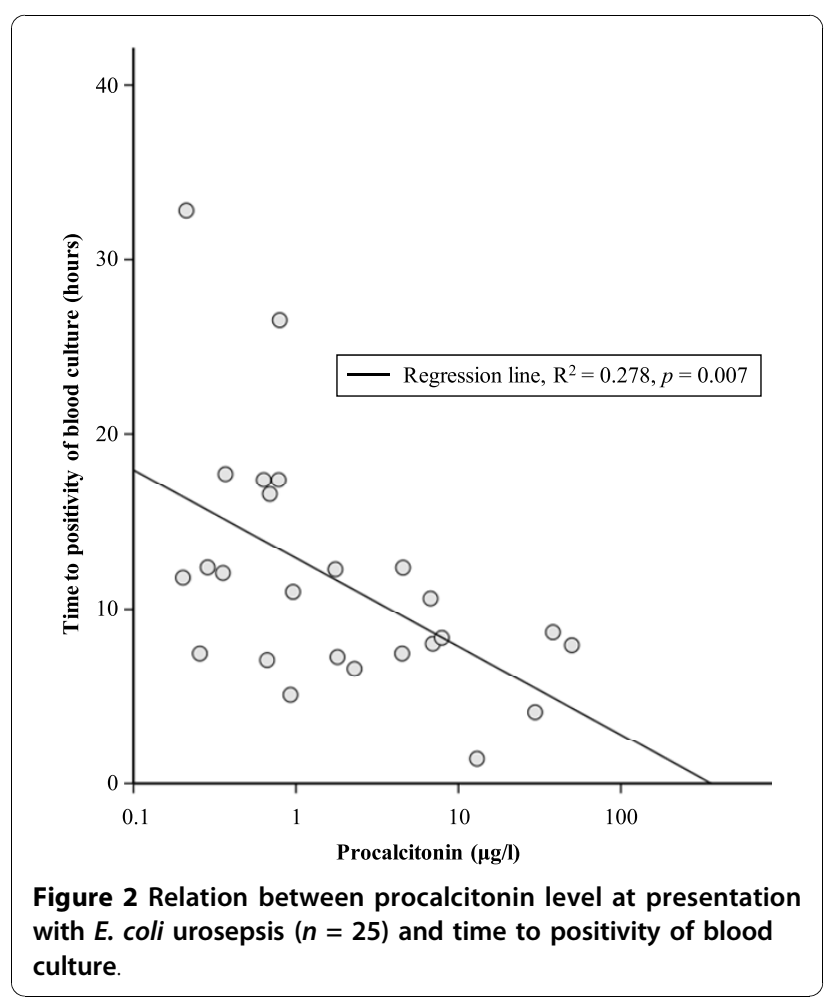


(median age 66 years) as various related coexisting illnesses might result in heterogeneous symptoms of bacteremia [27].

A relationship between PCT and TTP of the blood cultures has indirectly been suggested in the setting of discriminating blood contamination from bloodstream infection due to coagulase-negative staphylococci [28]. However, to our knowledge a direct relationship between PCT and the TTP of the blood culture in gram negative bacteremia has not been addressed previously. As the majority of bacteremic UTI is caused by gram negative microorganisms, we hypothesized that the bacterial load likely reflects the level of free lipopolysaccharide and thus the level of endotoxemia, which is correlated with the PCT value [18]. The TTP of the blood culture that depends on the rate of carbon dioxide production by the microorganisms can be used as a surrogate for systemic bacterial load, and we, thus, analyzed its correlation with PCT [29]. Because the TTP depends on the microorganism and the logistics around blood culture obtainment, we decided to analyze this for E. coli bacteremias of one center only [19]. We found a significant loglinear relationship between PCT value and TTP that supports biological plausibility between PCT value and the bacterial load of infection. Probably a similar phenomenon is indirectly illustrated by studies in lower respiratory tract infection that demonstrated that a low PCT value reflects a self-limiting disease that does not require antibiotic treatment while higher PCT values are associated with complicated outcome [30,31]. However, it should be emphasized that in this study low PCT levels were not indicative of absence of urinary tract infection. Hence, all patients included in this study received antimicrobial treatment. Therefore, additional studies are needed as to whether PCT might be of value in guiding antibiotic treatment of UTI and decision upon hospitalization as non-bacteremic patients are likely to be good candidates for outpatient treatment. In this respect, the results of a recent study are not promising as they do not support the use of PCT in helping guide physicians in deciding about hospitalization in patients with acute pyelonephritis [32]. This is in accordance with a smaller study demonstrating that PCT was not correlated with adverse outcome of acute pyelonephritis [33]. Interestingly, this latter study also showed significant higher PCT levels in bacteremic patients compared to nonbacteremic patients.

Our study has several strengths. First of all, we prospectively included consecutive patients with febrile UTI at multiple sites at primary care and ED setting. Thus, our study population reflects the broad population of routine clinical practice. Secondly, we were able to achieve blood culture and PCT results in over $90 \%$ of the study population. Furthermore, the rate of bacteremia was $23 \%$ indicating that many patients suffered the urosepsis syndrome [2]. Recommended by sepsis guidelines, all such patients require blood cultures before the initiation of antibiotic treatment [16]. Yet, using PCT $\leq 0.25 \mu \mathrm{g} / \mathrm{l}$ as a decision rule would have resulted in a $40 \%$ reduction of blood culture utilization, with $3 \%$ loss of detection of bacteremia. The relation between PCT and TTP supports previous suggestions in other infections that PCT may serve as a predictive biomarker for degree and severity of bacterial invasion.

There may, however, also be some limitations. Almost $30 \%$ of the patients did use antibiotics at the time of presentation as fever apparently developed during treatment of a nonfebrile UTI, for example, cystitis. This may have led to false negative blood cultures and could contribute to a relative low specificity of PCT in diagnosing bacteremia. However, antibiotic pretreatment for cystitis in The Netherlands usually concerns nitrofurantoin, a drug that is unlikely to affect bacteremia in UTI. Consistent herewith, pretreatment was associated with a higher chance of bacteremia and this suggests that antibiotic pretreatment did not skew our results towards negative blood cultures. Nevertheless, this still does not exclude the possibility that the rate of bacteremia may reflect an underestimate. Another limitation might be the measurement of PCT values that was done afterwards. Though the frozen storage of blood sample does not influence its PCT value, the measurement of PCT in routine clinical practice might be different [34]. Furthermore, when used to limit the use of blood cultures, a quick result of PCT, preferably by a readily available point-of-care assay, is mandatory for practical reasons.

This study might have consequences for the current practices on EDs as implementation of a PCT strategy likely is a cost-effective way to avoid taking blood cultures with a very low chance of yielding a positive culture. Moreover, besides in febrile UTI, this also seems to hold for patients presenting with community acquired pneumonia [26]. Taken together, these studies suggest that in the majority of patients presenting with febrile illnesses at ED, being either respiratory or urinary tract infections, medical diagnostic costs can be reduced. However, it should be highlighted that additional validation studies are needed, as the in- and exclusion criteria applied in this study might limit its generalizability to other settings and special patients groups. Furthermore, implementation studies addressing its cost-effectiveness are needed before the widespread use of PCT guidance on doing blood cultures in routine clinical practice can be recommended.

\section{Conclusions}

We conclude that PCT accurately predicts the presence of bacteremia and its bacterial load in adults with febrile 
UTI. A PCT value $\leq 0.25 \mu \mathrm{g} / \mathrm{l}$ sufficiently rules out bacteremia in febrile UTI and may be used to help guide efficient use of blood culture resources.

\section{Key messages}

- According to sepsis guidelines, blood cultures should be drawn to help diagnose bacteremia in case of febrile UTI, but the usefulness and cost-effectiveness of this practice have been questioned.

- This study confirms that bacteremia in febrile UTI can neither be predicted nor ruled out by bedside available clinical parameters.

- A low value $(\leq 0.25 \mu \mathrm{g} / \mathrm{l})$ of the biomarker procalcitonin (PCT) sufficiently rules out bacteremia in febrile UTI.

- Implementation of PCT into clinical practice with the aim to limit avoidable blood cultures is likely to be cost effective.

- In case of bacteremia the level of PCT appeared to be a marker of the bacterial load. Whether this might have implications for the dosage and length of antibiotic treatment awaits further studies.

\section{Additional material}

Additional file 1: Comparison of procalcitonin with C-reactive protein and erythrocyte sedimentation rate in predicting bacteremia in adults with febrile urinary tract infection. Results of a subset of patients with febrile UTI with additional laboratory values available.

\section{Abbreviations}

AUC: area under curve; CFU: colony forming unit; Cl: confidence interval; CRP: C-reactive protein; ED: emergency department; ESR: erythrocyte sedimentation rate; LR: likelihood ratio; NPV: negative predictive value; OR: odds ratio; PCT: procalcitonin; PPV: positive predictive value; $R O C$ : receiver operating characteristic; TTP: time to positivity; UTI: urinary tract infection.

\section{Acknowledgements}

The authors thank all the patients, medical personnel and the secretary staff of participating primary health care centers and emergency departments for their cooperation. We thank H. Nijzing (Brahms AG, Germany) for providing the Kryptor and PCT reagents. We are indebted to the clinical chemists $A$. Castel, P. Kok, G.L.A. Reijnierse, G.A.E Ponjee, M. Herruer, R.C. EijkmanRotteveel, P.W. Schenk and their personnel for their help in achievement and storage of the plasma samples. These data were presented in part at the $47^{\text {th }}$ Annual Meeting of the Infectious Diseases Society of America 2009, October 29 to November 1, Philadelphia, PA, Abstract LB-26. This study was partly supported by an unrestricted grant of the Bronovo Hospital Research Foundation.

\section{Author details}

'Department of Infectious Diseases, Leiden University Medical Center, Albinusdreef 2, 2333 ZA, Leiden, The Netherlands. ${ }^{2}$ Department of Internal Medicine, Bronovo Hospital, Bronovolaan 5, 2597 AX, The Hague, The Netherlands. ${ }^{3}$ Department of Medical Microbiology, Leiden University Medical Center, Albinusdreef 2, 2333 ZA, Leiden, The Netherlands.

${ }^{4}$ Department of Internal Medicine, Medical Center Haaglanden, Lijnbaan 32, 2512 VA, The Hague, The Netherlands. ${ }^{5}$ Department of Medical Microbiology, Bronovo Hospital, Bronovolaan 5, 2597 AX, The Hague, The Netherlands.
${ }^{6}$ Department of Internal Medicine, Groene Hart Hospital, Bleulandweg 10, $2803 \mathrm{HH}$, Gouda, The Netherlands. ${ }^{7}$ Department of Internal Medicine, Spaarne Hospital, Spaarnepoort 1, 2134 TM, Hoofddorp, The Netherlands. ${ }^{8}$ Department of Internal Medicine, Rijnland Hospital, Simon Smitweg 1, 2353 GA, Leiderdorp, The Netherlands. ${ }^{9}$ Department of Internal Medicine, Diaconessenhuis Leiden, Houtlaan 55, 2334 CK, Leiden, The Netherlands.

\section{Authors' contributions}

JWW, CN and JTD were responsible for the original design. CN, JWW and JTD were the guarantors. CN and TNB were responsible for data management, carried out the statistical analysis and wrote the initial draft supervised by JTD and JWW. CN, TNB, JWW, GHG, TK, GHWL, NMD, HCA and EMS were involved in patient recruitment and data collection. JWW, EJK, MJB, GHG, TK, GHWL, NMD, HCA and EMS critically revised the manuscript. All authors contributed to and approved the final version of the manuscript.

\section{Competing interests}

The authors declare that they have no competing interests.

Received: 17 May 2010 Revised: 8 July 2010

Accepted: 17 November 2010 Published: 17 November 2010

\section{References}

1. Pinson AG, Philbrick JT, Lindbeck GH, Schorling JB: Fever in the clinical diagnosis of acute pyelonephritis. Am J Emerg Med 1997, 15:148-151.

2. Kunin CM: Definition of acute pyelonephritis vs the urosepsis syndrome. Arch Intern Med 2003, 163:2393-2394.

3. Brown P, Ki M, Foxman B: Acute pyelonephritis among adults: cost of illness and considerations for the economic evaluation of therapy. Pharmacoeconomics 2005, 23:1123-1142.

4. Foxman B, Klemstine KL, Brown PD: Acute pyelonephritis in US hospitals in 1997: hospitalization and in-hospital mortality. Ann Epidemiol 2003, 13:144-150.

5. Jerkeman $\mathrm{M}, \mathrm{Braconier} \mathrm{JH}$ : Bacteremic and non-bacteremic febrile urinary tract infection-a review of 168 hospital-treated patients. Infection 1992, 20:143-145.

6. Leibovici L, Greenshtain S, Cohen O, Wysenbeek AJ: Toward improved empiric management of moderate to severe urinary tract infections. Arch Intern Med 1992, 152:2481-2486.

7. Hsu CY, Fang HC, Chou K, Chen CL, Lee PT, Chung HM: The clinical impact of bacteremia in complicated acute pyelonephritis. Am J Med Sci 2006, 332:175-180

8. Velasco M, Martínez JA, Moreno-Martínez A, Horcajada JP, Ruiz J, Barranco M, Almela M, Vila J, Mensa J: Blood cultures for women with uncomplicated acute pyelonephritis: are they necessary? Clin Infect Dis 2003, 37:1127-1130.

9. Chen Y, Nitzan O, Saliba W, Chazan B, Colodner R, Raz R: Are blood cultures necessary in the management of women with complicated pyelonephritis? J Infect 2006, 53:235-240.

10. van Nieuwkoop C, van't Wout JW, Spelt IC, Becker M, Kuijper EJ, Blom JW, Assendelft WJ, van Dissel JT: Prospective cohort study of acute pyelonephritis in adults: Safety of triage towards home based oral antimicrobial treatment. J Infect 2010, 60:114-121.

11. Bahagon $Y$, Raveh D, Schlesinger $Y$, Rudensky B, Yinnon AM: Prevalence and predictive features of bacteremic urinary tract infection in emergency department patients. Eur J Clin Microbiol Infect Dis 2007, 26:349-352.

12. Jerkeman $\mathrm{M}$, Braconier $\mathrm{JH}$ : Bacteremic and non-bacteremic febrile urinary tract infection-a review of 168 hospital-treated patients. Infection 1992, 20:143-145.

13. Lee $A$, Mirrett $S$, Reller $L B$, Weinstein MP: Detection of bloodstream infections in adults: how many blood cultures are needed? I Clin Microbiol 2007, 45:3546-3548.

14. Gander RM, Byrd L, DeCrescenzo M, Hirany S, Bowen M, Baughman J: Impact of blood cultures drawn by phlebotomy on contamination rates and health care costs in a hospital emergency department. J Clin Microbiol 2009, 47:1021-1024.

15. McCaig LF, McDonald LC, Cohen AL, Kuehnert MJ: Increasing blood culture use at US hospital emergency department visits, 2001 to 2004. Ann Emerg Med 2007, 50:42-48, 48.e1-2. 
16. Dellinger RP, Levy MM, Carlet JM, Bion J, Parker MM, Jaeschke R, Reinhart K, Angus DC, Brun-Buisson C, Beale R, Calandra T, Dhainaut JF, Gerlach H, Harvey M, Marini JJ, Marshall J, Ranieri M, Ramsay G, Sevransky J, Thompson BT, Townsend S, Vender JS, Zimmerman JL, Vincent JL, International Surviving Sepsis Campaign Guidelines Committee, American Association of Critical-Care Nurses, American College of Chest Physicians, American College of Emergency Physicians, Canadian Critical Care Society, European Society of Clinical Microbiology and Infectious Diseases, et al: Surviving Sepsis Campaign: international guidelines for management of severe sepsis and septic shock: 2008. Crit Care Med 2008, 36:296-327.

17. Jones AE, Fiechtl JF, Brown MD, Ballew JJ, Kline JA: Procalcitonin test in the diagnosis of bacteremia: a meta-analysis. Ann Emerg Med 2007, 50:34-41.

18. van Langevelde $\mathrm{P}$, Joop K, van Loon J, Frölich M, Groeneveld PH, Westendorp RG, van Dissel JT: Endotoxin, cytokines, and procalcitonin in febrile patients admitted to the hospital: identification of subjects at high risk of mortality. Clin Infect Dis 2000, 31:1343-1348.

19. Martínez JA, Pozo L, Almela M, Marco F, Soriano A, López F, Balasso V, Aguilar J, Mensa J: Microbial and clinical determinants of time-topositivity in patients with bacteraemia. Clin Microbiol Infect 2007, 13:709-716.

20. Wilson ML, Gaido L: Laboratory diagnosis of urinary tract infections in adult patients. Clin Infect Dis 2004, 38:1150-1158.

21. Sun GW, Shook TL, Kay GL: Inappropriate use of bivariable analysis to screen risk factors for use in multivariable analysis. J Clin Epidemiol 1996, 49:907-916.

22. van Nieuwkoop C, Bonten TN, Wout JW, Becker MJ, Groeneveld GH, Jansen $\mathrm{CL}$, van der Vorm ER, ljzerman EP, Rothbarth PH, TermeerVeringa EM, Kuijper EJ, van Dissel JT: Risk factors for bacteremia with uropathogen not cultured from urine in adults with febrile urinary tract infection. Clin Infect Dis 2010, 50:e69-e72.

23. Muller B, Schuetz $P$, Trampuz A: Circulating biomarkers as surrogates for bloodstream infections. Int J Antimicrob Agents 2007, 30:516-23.

24. Chirouze C, Schuhmacher H, Rabaud C, Gil H, Khayat N, Estavoyer JM, May $T$, Hoen B: Low serum procalcitonin level accurately predicts the absence of bacteremia in adult patients with acute fever. Clin Infect Dis 2002, 35:156-161.

25. Liaudat S, Dayer E, Praz G, Bille J, Troillet N: Usefulness of procalcitonin serum level for the diagnosis of bacteremia. Eur J Clin Microbiol Infect Dis 2001, 20:524-527.

26. Müller F, Christ-Crain M, Bregenzer T, Krause M, Zimmerli W, Mueller B, Schuetz P, ProHOSP Study Group: Procalcitonin levels Predict Bacteremia in Patients with community-acquired Pneumonia: A prospective cohort trial. Chest 2010, 138:121-129.

27. Fontanarosa PB, Kaeberlein FJ, Gerson LW, Thomson RB: Difficulty in predicting bacteremia in elderly emergency patients. Ann Emerg Med 1992, 21:842-848.

28. Schuetz P, Mueller B, Trampuz A: Serum procalcitonin for discrimination of blood contamination from bloodstream infection due to coagulasenegative staphylococci. Infection 2007, 35:352-355.

29. Blot F, Schmidt E, Nitenberg G, Tancrède C, Leclercq B, Laplanche A, Andremont A: Earlier positivity of central-venous- versus peripheralblood cultures is highly predictive of catheter-related sepsis. J Clin Microbiol 1998, 36:105-109.

30. Schuetz P, Christ-Crain M, Thomann R, Falconnier C, Wolbers M, Widmer I, Neidert S, Fricker T, Blum C, Schild U, Regez K, Schoenenberger R, Henzen C, Bregenzer T, Hoess C, Krause M, Bucher HC, Zimmerli W, Mueller B, ProHOSP Study Group: Effect of procalcitonin-based guidelines vs standard guidelines on antibiotic use in lower respiratory tract infections: the ProHOSP randomized controlled trial. JAMA 2009, 302:1059-1066.

31. Huang DT, Weissfeld LA, Kellum JA, Yealy DM, Kong L, Martino M, Angus DC, GenIMS Investigators: Risk prediction with procalcitonin and clinical rules in community-acquired pneumonia. Ann Emerg Med 2008, 52:48-58.

32. Claessens YE, Schmidt J, Batard E, Grabar S, Jegou D, Hausfater P, Kierzek G, Guérin S, Pourriat JL, Dhainaut JF, Ginsburg C, BIS Study Group: Can Creactive protein, procalcitonin and mid-regional pro-atrial natriuretic peptide measurements guide choice of in-patient or out-patient care in acute pyelonephritis? Biomarkers In Sepsis (BIS) multicentre study. Clin Microbiol Infect 2010, 16:753-760.
33. Lemiale V, Renaud B, Moutereau S, N'Gako A, Salloum M, Calmettes MJ, Hervé J, Boraud C, Santin A, Grégo JC, Braconnier F, Roupie E: A single procalcitonin level does not predict adverse outcomes of women with pyelonephritis. Eur Urol 2007, 51:1394-1401.

34. Schuetz $P$, Christ-Crain M, Huber AR, Muller B: Long-term stability of procalcitonin in frozen samples and comparison of Kryptor and VIDAS automated immunoassays. Clin Biochem 2010, 43:341-344.

\section{doi:10.1186/cc9328}

Cite this article as: van Nieuwkoop et al:: Procalcitonin reflects bacteremia and bacterial load in urosepsis syndrome: a prospective observational study. Critical Care 2010 14:R206.

\section{Submit your next manuscript to BioMed Central and take full advantage of:}

- Convenient online submission

- Thorough peer review

- No space constraints or color figure charges

- Immediate publication on acceptance

- Inclusion in PubMed, CAS, Scopus and Google Scholar

- Research which is freely available for redistribution

Submit your manuscript at www.biomedcentral.com/submit
Biomed Central 